\title{
QTc prolongation in COVID-19 Patients: Pathophysiology and Considerations for Prevention and Treatment
}

\author{
Neil Gupta, Zaid Al-Jebaje* and Valentina Kutyifa \\ University of Rochester, Medical Center, Rochester, USA \\ *Corresponding author: Dr. Zaid Al Jebaje, Fellow of Cardiovascular Medicine, University of Rochester, Department on \\ Medicine, Division of Cardiology, Rochester, NY, 601 Elmwood Avenue, Rochester, NY 14642, USA
}

\section{ARTICLE INFO}

Received: 幽 August 10, 2020

Published: 慧 August 25, 2020

Citation: Neil Gupta, Zaid Al-Jebaje, Valentina Kutyifa. QTc prolongation in COVID-19 Patients: Pathophysiology and Considerations for Prevention and Treatment. Biomed J Sci \& Tech Res 29(4)-2020. BJSTR. MS.ID.004848.

\begin{abstract}
Severe acute respiratory syndrome coronavirus 2 (SARS-CoV-2) causing coronavirus disease 2019 (COVID- 19) has had large implications in patients with preexisting cardiovascular conditions. COVID-19 has a high inflammatory burden which places patients at risk for developing repolarization changes with resultant prolongation of the QT syndrome and Torsades de Pointes (TdP). This is potentially further exacerbated by medications being used to treat COVID-19. However, there have been no cases of TdP reported without a specific link to medication during a COVID-19 infection.
\end{abstract}

Abbreviations: ARDS: Acute Respiratory Distress Syndrome; ICU: Intensive Care Unit; CVD: cardiovascular disease; ACE2: Angiotensin-Converting Enzyme 2; CRS: Cytokine Release Syndrome; LQTS: Long QT Syndrome; SARS: Severe Acute Respiratory Syndrome; HERG: Human Ether- a-go-go-Related Gene

\section{Case Report}

\section{History of Presentation}

A 46-year-old male was admitted to the hospital for an episode of syncope from non-infectious diarrhea. An electrocardiogram at that time showed normal sinus rhythm with a QTc of $419 \mathrm{~ms}$ (Figure 1). During his hospital admission he developed a fever of 102 degrees with a chest x-ray concerning for pneumonia and was subsequently found to be positive for SARS-CoV-2. He was stable and discharged home with instructions to self-isolate. Five days later he presented to the Emergency Department with complaints of light-headedness with exertion and hypoxia with an oxygen saturation of $51 \%$ on room air. He was quickly intubated for hypoxic respiratory failure and started on peripheral vasopressors for persistent hypotension. An arterial blood gas indicated severe acute respiratory distress syndrome (ARDS), prompting paralyzation and proning. He received initial treatment with azithromycin $500 \mathrm{mg}$ for three days and piperacillin/tazobactam for presumed concomitant pneumonia. His hospital course was complicated by prolonged ARDS and delirium requiring Quetiapine. Given his prolonged Intensive Care Unit (ICU) admission he was started on Remdesivir on day 13 of hospitalization. On day 16, he developed pulseless polymorphic ventricular tachycardia.

\section{Past Medical History}

The patient had a history of hypertension that was treated with amlodipine $10 \mathrm{mg}$ daily and labetalol $200 \mathrm{mg}$ twice a day

\section{Differential Diagnosis}

The patient had severe sepsis and hypoxic respiratory failure from COVID-19

\section{Management}

Cardiopulmonary resuscitation was initiated immediately with administration of $300 \mathrm{mg}$ of amiodarone and external defibrillation before achieving spontaneous return of circulation. His electrolytes were stable prior to his cardiac arrest. His Quetiapine was discontinued, and he was not on any other concomitant QT 
prolonging medications except for as needed haloperidol. An electrocardiogram after his arrest revealed a QTc of 441 ms with no ischemic changes (Figure 2). Electrophysiology was consulted who recommended tapering amiodarone and obtaining an echocardiogram which showed normal biventricular function and no wall motion abnormalities.

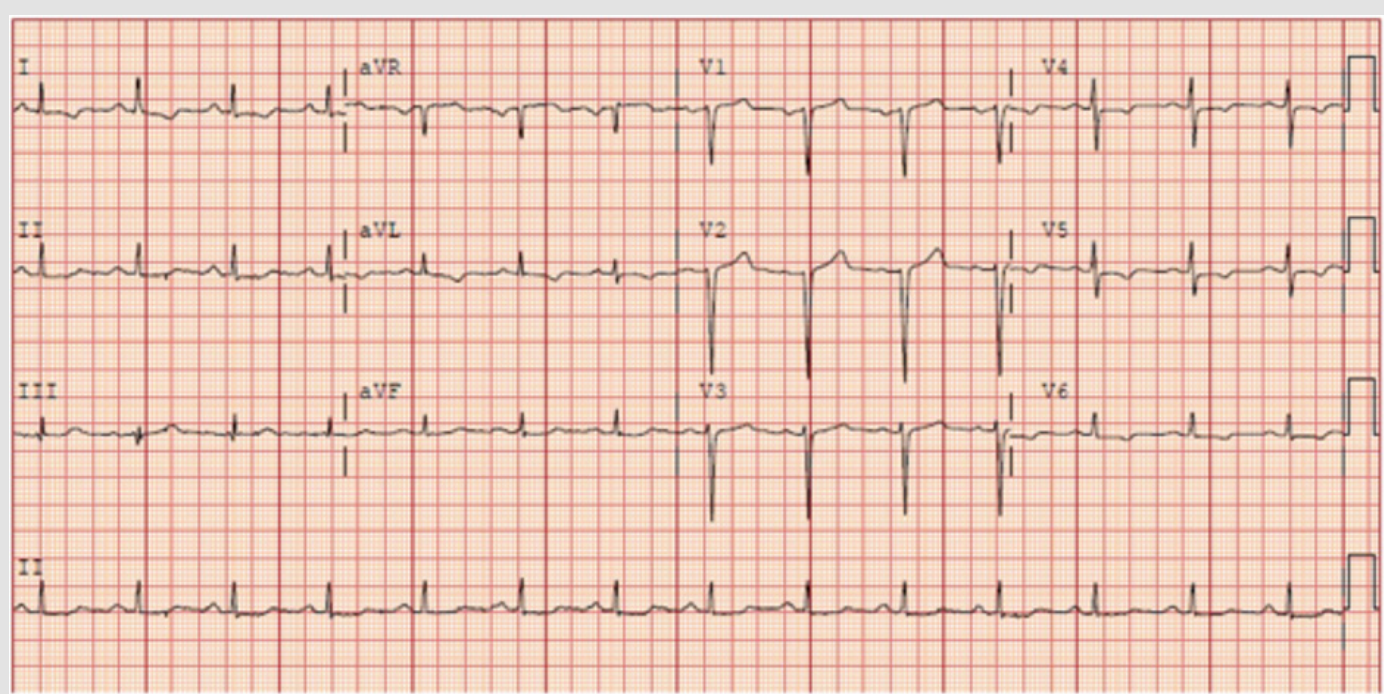

Figure 1: EKG on presentation.

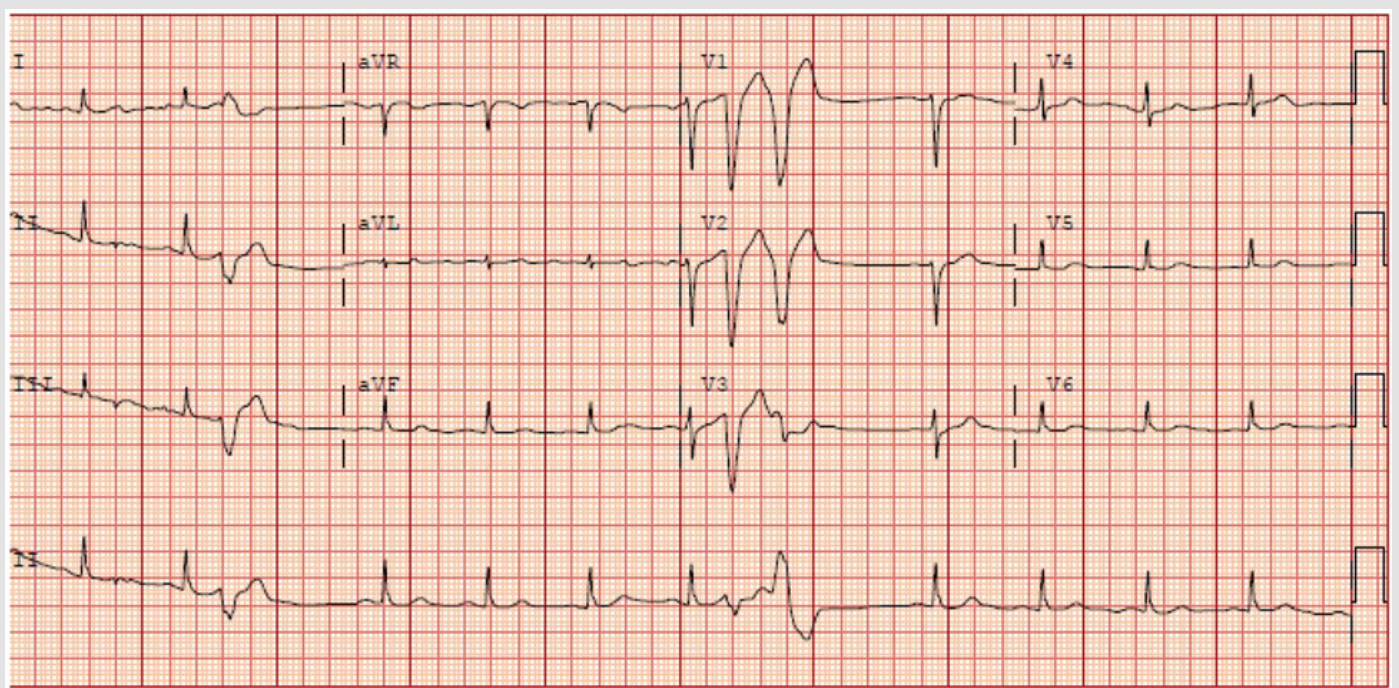

Figure 2: post arrest EKG.

\section{Follow-Up}

Our patient remained in normal sinus rhythm throughout his hospitalization and was transferred out of the ICU to the medical floors where he still resides. He is undergoing evaluation for inpatient rehabilitation.

\section{Discussion}

SARS-CoV-2 is the underlying virus causing the global pandemic known as COVID-19. Patients with preexisting cardiovascular disease (CVD) who contract SARS-CoV-2 appear to have an increased risk of severe disease, morbidity and mortality. Furthermore, infection with SARS-CoV-2 has been associated with myocardial injury, defined as an increased serum troponin level, and arrhythmias $[1,2]$. The exact pathophysiology of myocardial injury has not yet been determined. Studies have shown that the SARS-CoV-2 virus uses the angiotensin-converting enzyme 2 (ACE2) protein for entry into cells [1]. ACE2 is a membrane-bound aminopeptidase found predominantly in cardiac and pulmonary alveolar cells. Viral entry into cardiac myocytes may be directly related to the ACE2 protein leading to myocardial injury [3]. Myocardial injury likely increases the risk of developing arrhythmias which represented a leading complication (19.6\%) in a study of 138 hospitalized COVID-19 patients. Patients with COVID-19 who have myocardial injury have an increased risk of developing ventricular arrhythmias [1]. 
It is important to recognize that myocardial injury may play a role in the development of arrhythmias in COVID-19 patients. However, the development of malignant ventricular arrhythmias like TdP is likely related to the prolongation of the QT interval in patients with a large immunological response or those receiving QT prolonging medications. SARS-CoV-2 causes an exaggerated immune response leading to cytokine release syndrome (CRS) leading to the continuous activation of lymphocytes and macrophages that release cytokines. These high levels of cytokines can lead to apoptosis or necrosis of cardiac myocytes. Furthermore, strong evidence supports that high levels of inflammation is a risk factor for the development of acquired long QT syndrome (LQTS) [4]. Specifically increased levels of interleukin-6 (IL-6) have been seen in COVID-19 patients. IL-6 plays an important role in the large release of cytokines and is not only present at high levels in patients with SARS- CoV-2 but also in patients with Severe Acute Respiratory Syndrome (SARS) and Middle East Respiratory Syndrome (MERS-CoV), other coronaviruses [5]. IL-6 has been associated with acquired LQTS and increased risk of developing polymorphic ventricular tachycardia like TdP. In cases of acquired LQTS the human ether- a-go-go-related gene (hERG) encodes the rapid component potassium channel that plays a critical role in cardiac repolarization [6]. IL-6 has been proven to negatively modulate the hERG channel thus prolonging action potential duration in ventricular myocytes. IL-6 also inhibits cytochrome p450 which can increase the bioavailability of several medications including those known to prolong the QT interval, like azithromycin which is being used to treat COVID-19 patients [4].

Several medications being used to treat COVID-19 including hydroxychloroquine, chloroquine and azithromycin are known to be QT prolonging agents and increase a patient's risk of developing acquired LQTS. Although the risk is low, it is likely exacerbated by elevated levels of IL- 6 and the use of other sedative medications. A recent cohort study showed that patients who received hydroxychloroquine for COVID-19 were at high risk of QTc prolongation and concurrent treatment with azithromycin was associated with greater changes in QTc [7]. Similar findings were seen in 40 ICU patients treated with hydroxychloroquine with or without azithromycin with larger increases in QTc in the group treated with azithromycin [8,9]. Hydroxychloroquine, chloroquine and azithromycin are known to prolong QTc however little is known about Remdesivir and its effects on the QT interval. It is certainly possible that Remdesivir contributed to TdP in our patient, however, it remains unclear. Measures must be used to decrease the incidence of TdP in COVID-19. In a case study from Mitra, intravenous lidocaine was used to successfully shorten the QTc interval from $620 \mathrm{~ms}$ to $550 \mathrm{~ms} .9$ It may be beneficial as well to block the IL-6 pathway with an anti-IL-6 receptor monoclonal- antibody like tocilizumab which has shortened QTc in patients with rheumatoid arthritis [4].

\section{Conclusion}

Factors that contribute to the development of LQTS from a hyperacute immune response with CRS and medications known to prolong the QT interval should all be taken into account when trying to prevent TdP in COVID-19 patients. Ultimately, QTc should be monitored with these patients to help prevent TdP and, if experimental drugs prove efficacious, methods should be implemented to help shorten the QTc if it is prolonged.

\section{Learning Objective}

I. The large inflammatory response in COVID-19 patients makes them more susceptible to acquiring LQTS and TdP

II. Experimental medications for the treatment of COVID-19 should be used with caution as they increase the risk of LQTS and TdP

\section{References}

1. Elissa Driggin, Mahesh V Madhavan, Behnood Bikdeli, Taylor Chuich, Justin Laracy, et al. (2020) Cardiovascular Considerations for Patients, Health Care Workers, and Health Systems During the Coronavirus Disease 2019 (COVID-19) Pandemic. J Am Coll Cardiol 75(8).

2. Shaobo Shi, Mu Qin, Bo Shen, Yuli Cai, Tao Liu, et al. (2020) Association of Cardiac Injury With Mortality in Hospitalized Patients With COVID-19 in Wuhan, China. JAMA Cardiol.

3. Mehra MR, Desai SS, Kuy S, Henry TD, Patel AN (2020) Cardiovascular Disease, Drug Therapy, and Mortality in Covid-19. N Engl J Med.

4. Lazzerini PE, Boutjdir M, Capecchi PL (2020) COVID-19, Arrhythmic Risk and Inflammation: Mind the Gap! Circulation 2020 142(7-9).

5. Zhang C, Wu Z, Li JW, Zhao H, Wang GQ (2020) The cytokine release syndrome (CRS) of severe COVID-19 and Interleukin-6 receptor (IL6R) antagonist Tocilizumab may be the key to reduce the mortality. Int J Antimicrob Agents 2020: 105954.

6. Ademuyiwa S Aromolaran, Ujala Srivastava, Alessandra Alí, Mohamed Chahine, Deana Lazaro, et al. (2018) Interleukin-6 inhibition of hERG underlies risk for acquired long QT in cardiac and systemic inflammation. PLoS One 13: e0208321.

7. Nicholas J Mercuro, Christina F Yen, David J Shim, Timothy R Maher, Christopher M Mc Coy, et al. (2019) Risk of QT Interval Prolongation Associated With Use of Hydroxychloroquine With or Without Concomitant Azithromycin Among Hospitalized Patients Testing Positive for Coronavirus Disease 2019 (COVID-19). JAMA Cardiol 2020.

8. Francis Bessière, Hugo Roccia, Antoine Delinière, Rome Charrière, Philippe Chevalier, et al. (2020) Assessment of QT Intervals in a Case Series of Patients With Coronavirus Disease 2019 (COVID-19) Infection Treated With Hydroxychloroquine Alone or in Combination With Azithromycin in an Intensive Care Unit. JAMA Cardiol 2020.

9. Mitra RL, Greenstein SA, Epstein LM (2020) An algorithm for managing QT prolongation in coronavirus disease 2019 (COVID-19) patients treated with either chloroquine or hydroxychloroquine in conjunction with azithromycin: Possible benefits of intravenous lidocaine. HeartRhythm Case Rep 2020 6(5): 244-248. 
ISSN: 2574-1241

DOI: $10.26717 /$ BJSTR.2020.29.004848

Zaid Al-Jebaje. Biomed J Sci \& Tech Res

(C) This work is licensed under Creative

Submission Link: https://biomedres.us/submit-manuscript.php

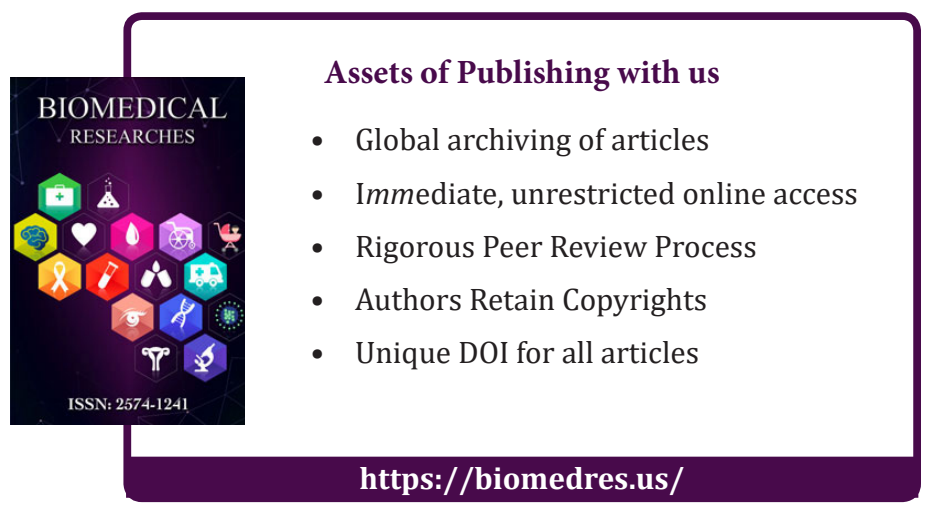

